\title{
Representations and Derivations of Modules
}

\author{
JANKO BRAČIČ
}

\begin{abstract}
In this article we define and study derivations between bimodules. In particular, we define the Gelfand radical of a Banach bimodule and show that, under some reasonable conditions, every derivation between two Banach bimodules over a commutative Banach algebra maps into the Gelfand radical. This is a module version of the famous Singer-Wermer Theorem.
\end{abstract}

\section{INTRODUCTION}

Let $\mathcal{A}$ be an algebra and $\mathcal{X}$ an $\mathcal{A}$-bimodule. If $\delta$ is a derivation from $\mathcal{A}$ into $X$, then its adjoint $\delta^{\prime}: X^{\prime} \rightarrow \mathcal{A}^{\prime}$ is, in general, no longer a derivation. The situation is rather different if we consider $\delta^{\prime \prime}: \mathcal{A}^{\prime \prime} \rightarrow X^{\prime \prime}$, see [5]. The aim of this article is to extend the notion of derivation to bimodules in such a way that the adjoint of a derivation is a derivation between the dual bimodules.

Our starting point is the identity

$$
\delta(a b)=a \cdot \delta(b)+\delta(a) \cdot b \quad(a, b \in \mathcal{A}),
$$

written in the form

$$
\delta(a b)=\kappa(a) \delta(b)+\chi(b) \delta(a) \quad(a, b \in \mathcal{A}) .
$$

Here $\kappa$ and $\chi$ are the representation, respectively the antirepresentation, associated with the $\mathcal{A}$-bimodule $\mathcal{X}$. In Section 2 we study those module homomorphisms (resp. antihomomorphisms) that can be called representations (resp. antirepresentations) of modules. Then, in Section 3, we define derivations between bimodules by rewriting (2) in the context of bimodules. It is also shown there that the adjoint of a derivation between two bimodules is again a derivation. In

2000 Mathematics Subject Classification. 46H25, 46H15, $47 \mathrm{~B} 47$.

Key words and phrases. Module, Banach module, representation, derivation, Gleason-Kahane-Żelazko Theorem, Singer-Wermer Theorem. 
Section 4 we confine ourselves to the case of Banach bimodules over a unital commutative Banach algebra. We introduce the notion of point multiplier and define the Gelfand radical of a Banach bimodule. The last result in the section is a variant of the Singer-Wermer Theorem.

The notation we shall use through this article is quite standard. If $X$ is a linear space, then $X^{\prime}$ denotes its dual. Topological dual of a Banach space $X$ is $X^{*}$. The space of all linear maps from a linear space $X$ into a linear space $y$ is denoted by $L(X, y)$ and $L(X)$ denotes the algebra of all linear operators on $X$. For $T \in L(X, y)$ let $T^{\prime} \in L\left(\mathcal{Y}^{\prime}, X^{\prime}\right)$ denote its adjoint map. In the case of Banach spaces $B(X, Y)$ denotes the Banach space of all bounded linear maps, $B(X)$ is the Banach algebra of all bounded linear operators on $\mathcal{X}$, and $T^{*} \in B\left(y^{*}, X^{*}\right)$ is the topological adjoint of $T \in B(X, y)$.

Let $X$ and $y$ be left $\mathcal{A}$-modules. We shall denote by $L_{\mathcal{A}}(X, y)$ the space of all module homomorphisms from $X$ into $y$. In the case of Banach left $\mathcal{A}$-modules, $B_{\mathcal{A}}(\mathcal{X}, y)$ denotes the Banach space of all bounded module homomorphisms. Of course, $L(X, y)_{\mathcal{B}}, B(X, y)_{\mathcal{B}}$, etc., have similar meaning. If $\mathcal{X}=\mathcal{Y}$, then we shall write $L_{\mathcal{A}}(\mathcal{X})$, etc.

\section{Representations}

It is assumed that the reader is familiar with the notion of representation (and antirepresentation) of an algebra. At this point we would like to point out only two things.

If $\theta$ is a representation of an algebra $\mathcal{A}$ on a space $\mathcal{X}$, then it defines a left $\mathcal{A}$-module structure on $\mathcal{X}$. Conversely, if $\mathcal{X}$ is a left $\mathcal{A}$-module, then the map $\theta: \mathcal{A} \rightarrow L(\mathcal{X})$ defined by $\theta(a) x=a \cdot x \quad(a \in \mathcal{A}, x \in \mathcal{X})$ is a representation of $\mathcal{A}$ on $\mathcal{X}$. In this situation we shall say that $\theta$ is the representation associated to the module $\mathcal{X}$. There is a similar relationship between antirepresentations and right modules. In the case of Banach modules representations and antirepresentations are normed (i.e. they map into $B(X))$ and bounded. The reader is referred to $[2,4,6]$ for details.

Let $y$ or $z$ be an $\mathcal{A}$-module. Then $L(y, z)$ has a natural structure of an $\mathcal{A}$-module, see [4], Definition 1.4.12. For instance, if $Z$ is a left $\mathcal{A}$-module, then $L(y, z)$ is a left $\mathcal{A}$-module via the multiplication

$$
(a \cdot T) y=a \cdot T y \quad(a \in \mathcal{A}, T \in L(y, z), y \in y) .
$$

In the case of Banach modules $y$ and $z$ the space $B(y, z)$ can be given a structure of a Banach module. 
Let $\mathcal{X}$ be a left $\mathcal{A}$-module. We shall say that a linear map $\Theta: X \rightarrow$ $L(\mathcal{Y}, z)$ is a representation of $\mathcal{X}$ on a pair $(y, z)$ if there exists a representation $\theta$ of the algebra $\mathcal{A}$ on $z$ such that

$$
\Theta(a \cdot x)=\theta(a) \Theta(x) \quad(a \in \mathcal{A}, x \in \mathcal{X}) .
$$

An antirepresentation of $\mathcal{X}$ on a pair $(\mathcal{Y}, Z)$ is a linear map $\mathrm{H}: \mathcal{X} \rightarrow$ $L(y, z)$ such that

$$
\mathrm{H}(a \cdot x)=\mathrm{H}(x) \eta(a) \quad(a \in \mathcal{A}, x \in \mathcal{X})
$$

for some antirepresentation $\eta$ of the algebra $\mathcal{A}$ on $y$.

Now let $\mathcal{X}$ be an $\mathcal{A}$ - $\mathcal{B}$-bimodule. A linear map $\Theta: X \rightarrow L(\mathcal{Y}, z)$ is a representation of $\mathcal{X}$ on a pair $(\mathcal{Y}, Z)$ if it represents both module structures of $X$ on $(y, Z)$. Thus, $\Theta$ is a representation if there exist representations $\theta: \mathcal{A} \rightarrow L(\mathcal{Z})$ and $\pi: \mathcal{B} \rightarrow L(y)$ such that

$$
\Theta(a \cdot x \cdot b)=\theta(a) \Theta(x) \pi(b) \quad(a \in \mathcal{A}, x \in \mathcal{X}, b \in \mathcal{B}) .
$$

Similarly, a linear map $\mathrm{H}: \mathcal{X} \rightarrow L(\mathcal{y}, \mathcal{Z})$ is an antirepresentation if there exist antirepresentations $\eta: \mathcal{A} \rightarrow L(y)$ and $\sigma: \mathcal{B} \rightarrow L(z)$ such that

$$
\mathrm{H}(a \cdot x \cdot b)=\sigma(b) \mathrm{H}(x) \eta(a) \quad(a \in \mathcal{A}, x \in \mathcal{X}, b \in \mathcal{B}) .
$$

Example 2.1. Let $\mathcal{X}$ be an $\mathcal{A}$-bimodule with the associated representation $\kappa$ and antirepresentation $\chi$. For each $x \in \mathcal{X}$ define maps $\Lambda(x)$ and $\mathrm{P}(x)$ from $\mathcal{A}$ into $\mathcal{X}$ by

$$
\Lambda(x): a \mapsto x \cdot a \quad \text { and } \mathrm{P}(x): a \mapsto a \cdot x \quad(a \in \mathcal{A}) .
$$

Let $\lambda: \mathcal{A} \rightarrow L(\mathcal{A})$ be the left regular representation and $\rho: \mathcal{A} \rightarrow$ $L(\mathcal{A})$ the right regular representation of $\mathcal{A}$. Then

$$
\Lambda(a \cdot x \cdot b)=\kappa(a) \Lambda(x) \lambda(b) \quad(a, b \in \mathcal{A}, x \in \mathcal{X})
$$

and

$$
\mathrm{P}(a \cdot x \cdot b)=\chi(b) \mathrm{P}(x) \rho(a) \quad(a, b \in \mathcal{A}, x \in \mathcal{X}) .
$$

Thus $\Lambda$ is a representation and $\mathrm{P}$ is an antirepresentation of $\mathcal{X}$ on the pair $(\mathcal{A}, X)$. We shall call $\Lambda$ the left regular representation of $\mathcal{X}$ and $\mathrm{P}$ the right regular representation of $\mathcal{X}$.

Note that a left (respectively, a right) module has only the right (respectively, the left) regular representation.

Let $\Theta$ be a representation of a left $\mathcal{A}$-module $\mathcal{X}$ on a pair $(\mathcal{Y}, Z)$. We shall say that a representation $\theta$ of the algebra $\mathcal{A}$ on $\mathcal{Z}$ corresponds to $\Theta$ if $\Theta(a \cdot x)=\theta(a) \Theta(x) \quad(a \in \mathcal{A}, x \in \mathcal{X})$. In general a representation $\theta$ is not uniquely determined by $\Theta$. For instance, if $\Theta$ 
is trivial (i.e. $\Theta(x)=0$ for all $x \in \mathcal{X}$ ), then any representation of $\mathcal{A}$ on $Z$ corresponds to $\Theta$.

Proposition 2.2. Let $\mathcal{X}$ be a left $\mathcal{A}$-module. If $\Theta$ is a representation of $\mathcal{X}$ on a pair $(\mathcal{Y}, Z)$ such that

$$
\operatorname{lin}\left\{\bigcup_{x \in \mathcal{X}} \operatorname{im} \Theta(x)\right\}=z
$$

then there exists only one representation of $\mathcal{A}$ on $\mathcal{Z}$ that corresponds to $\Theta$.

For an antirepresentation $\mathrm{H}$ of $\mathcal{X}$ on a pair $(\mathcal{Y}, Z)$ there exists only one corresponding antirepresentation of $\mathcal{A}$ on $y$ if

$$
\bigcap_{x \in \mathcal{X}} \operatorname{ker} \mathrm{H}(x)=\{0\}
$$

We shall omit the proof since it is evident.

The left and the right module structures on an $\mathcal{A}$ - $\mathcal{B}$-bimodule $X$ are connected by the equation

$$
(a \cdot x) \cdot b=a \cdot(x \cdot b) \quad(a \in \mathcal{A}, x \in \mathcal{X}, b \in \mathcal{B}) .
$$

This property of bimodules is very important. For instance, let $y$ be a left and a right $\mathcal{A}$-module such that the module structures are not connected by the equation (3). Then derivations from $\mathcal{A}$ into $y$ cannot be well defined. Namely, for some $a, b, c \in \mathcal{A}$, the vectors $\delta((a b) c)$ and $\delta(a(b c))$, where $\delta: \mathcal{A} \rightarrow \mathrm{y}$ is a linear map satisfying (1), can be, in general, distinct.

Since our goal is to define a derivations between bimodules, we need a property which is a counterpart of (3) in the context of modules.

Note that if $\kappa: \mathcal{A} \rightarrow L(\mathcal{X})$, respectively $\chi: \mathcal{B} \rightarrow L(X)$, are the associated representation of $\mathcal{A}$, respectively the associated antirepresentation of $\mathcal{B}$, then $(3)$ can be interpreted as

$$
\kappa(a) \in L(\mathcal{X})_{\mathcal{B}} \quad \text { and } \quad \chi(b) \in L_{\mathcal{A}}(\mathcal{X}) \quad(a \in \mathcal{A}, b \in \mathcal{B}) .
$$

Definition 2.3. Let $X$ be a left $\mathcal{A}$-module and $\Theta$ a representation of $\mathcal{X}$ on a pair $(\mathcal{y}, \mathcal{Z})$. Assume that $\mathcal{Y}$ and $\mathcal{Z}$ are left $\mathcal{B}$-modules. Then $\Theta$ is said to be left $\mathcal{B}$-modular if

$$
\Theta(x) \in L_{\mathcal{B}}(y, z) \quad(x \in X),
$$

i.e. if, for each $x \in \mathcal{X}$, the map $\Theta(x)$ is a module homomorphism between left $\mathcal{B}$-modules $y$ and $z$. 
Right modularity and modularity of antirepresentations are defined similarly.

Examples 2.4. (a) Let $\mathcal{X}$ be an $\mathcal{A}$-bimodule. Then the left regular representation $\Lambda$ is right $\mathcal{A}$-modular:

$$
\begin{gathered}
\Lambda(x)\left(a_{1} a_{2}\right)=x \cdot\left(a_{1} a_{2}\right)=\left(x \cdot a_{1}\right) \cdot a_{2}=\left[\Lambda(x) a_{1}\right] \cdot a_{2} \\
\left(a_{1}, a_{2} \in \mathcal{A}, x \in \mathcal{X}\right) .
\end{gathered}
$$

Similarly, the right regular representation $\mathrm{P}$ is left $\mathcal{A}$-modular.

(b) Let $\mathcal{X}$ be a left $\mathcal{A}$-module and $\mathcal{Y}$ a right $\mathcal{B}$-module. Then the tensor product $\mathcal{X} \otimes \mathcal{Y}$ is an $\mathcal{A}$ - $\mathcal{B}$-bimodule (see [4], Example 1.4.9 (ii)). It is easily seen that

$$
\Theta(x) y:=x \otimes y \quad(x \in \mathcal{X}, y \in \mathcal{y})
$$

defines a right $\mathcal{B}$-modular representation of $\mathcal{X}$ on $(y, X \otimes y)$.

Definition 2.5. Let $X$ be an $\mathcal{A}$-bimodule and let it be represented on a pair of linear spaces $(\mathcal{y}, \mathcal{Z})$ by a representation $\Theta$ and by an antirepresentation $\mathrm{H}$. Then $(\boldsymbol{y}, \boldsymbol{z})$ is said to be a modular pair over $X$ if $\Theta$ is right $\mathcal{A}$-modular and $\mathrm{H}$ is left $\mathcal{A}$-modular.

In the case of Banach modules it is also required that $\Theta$ and $\mathrm{H}$ are bounded.

Examples 2.6. (a) If an $\mathcal{A}$-bimodule $X$ is represented on $(\mathcal{A}, X)$ via the regular representations $\Lambda$ and $\mathrm{P}$, then the pair $(\mathcal{A}, \mathcal{X})$ is modular over $X$.

(b) Let $\mathcal{X}$ be an $\mathcal{A}$-bimodule. Then $\mathcal{X} \otimes_{\mathcal{A}} \mathcal{X}$ is also an $\mathcal{A}$-bimodule (see [4], Example 1.4.9 (iii)). For each $x \in \mathcal{X}$ define maps $\Theta(x)$ and $\mathrm{H}(x)$ from $\mathcal{X}$ into $X \otimes_{\mathcal{A}} \mathcal{X}$ by

$$
\Theta(x) y:=x \otimes y \quad \text { and } \quad \mathrm{H}(x) y:=y \otimes x \quad(y \in \mathcal{X}) .
$$

It is straightforward to see that $\Theta$ is a right $\mathcal{A}$-modular representation and $\mathrm{H}$ is a left $\mathcal{A}$-modular antirepresentation of the bimodule $\mathcal{X}$ on the pair $\left(X, X \otimes_{\mathcal{A}} X\right)$.

\section{DeRivations}

Let $(y, z)$ be a modular pair over $X$. Denote by $\Theta: X \rightarrow L(y, z)_{\mathcal{A}}$ the corresponding representation and let $\theta: \mathcal{A} \rightarrow L(Z)$ and $\pi: \mathcal{A} \rightarrow$ $L(y)$ be the associated representations of the algebra $\mathcal{A}$. By $\mathrm{H}: \mathcal{X} \rightarrow$ $L_{\mathcal{A}}(\mathcal{Y}, Z)$ denote the corresponding antirepresentation of $X$ and let $\sigma: \mathcal{A} \rightarrow L(z)$ and $\eta: \mathcal{A} \rightarrow L(y)$ be the associated antirepresentations of the algebra $\mathcal{A}$. 
Definition 3.1. A linear map $\Delta: X \rightarrow Z$ is a derivation if there exists an ordinary derivation $\delta: \mathcal{A} \rightarrow y$ such that

$$
\Delta(a \cdot x)=\theta(a) \Delta(x)+\mathrm{H}(x) \delta(a)=a \cdot \Delta(x)+\mathrm{H}(x) \delta(a)
$$

and

$$
\Delta(x \cdot a)=\Theta(x) \delta(a)+\sigma(a) \Delta(x)=\Theta(x) \delta(a)+\Delta(x) \cdot a
$$

for all $a \in \mathcal{A}$ and all $x \in \mathcal{X}$.

Note that $\Delta$ is well defined because of the modularity of $\Theta$ and $H$.

If $\Delta$ is a derivation from $X$ into $Z$, then it is not necessarily true that there exists only one ordinary derivation $\delta: \mathcal{A} \rightarrow y$ such that the equations in Definition 3.1 are fulfilled. However, if $\Theta$ and $\mathrm{H}$ are nondegenerate, i.e.

$$
\bigcap_{x \in X} \operatorname{ker} \Theta(x)=\{0\}=\bigcap_{x \in \mathcal{X}} \operatorname{ker} \mathrm{H}(x),
$$

then there is a uniquely determined derivation $\delta$ which corresponds to $\Delta$. From now on we shall always assume that $\Theta$ and $\mathrm{H}$ are nondegenerate.

Examples 3.2. (a) Every ordinary derivation $\delta: \mathcal{A} \rightarrow X$ is a derivation in the sense of Definition 3.1. Indeed, the pair $(X, X)$ is modular over $\mathcal{A}$ and $\delta$ corresponds to itself.

(b) If $(y, z)$ is a modular pair over $X$, then every bimodule homomorphism $\mathrm{M} \in L_{\mathcal{A}}(X, Z)_{\mathcal{A}}$ is a derivation. The corresponding ordinary derivation from $\mathcal{A}$ into $y$ is the trivial one. On the other hand, if $\Delta: X \rightarrow Z$ is a derivation with 0 as the corresponding ordinary derivation, then $\Delta$ is a module homomorphism.

(c) Let $(y, z)$ be a modular pair over $X$. For $y \in \mathcal{y}$ define

$$
\Delta_{y}: x \mapsto \Theta(x) y-\mathrm{H}(x) y \quad(x \in \mathcal{X}) .
$$

This is an inner derivation from $\mathcal{X}$ into $\mathcal{Z}$. The corresponding ordinary derivation is the inner derivation

$$
\delta_{y}: a \mapsto a \cdot y-y \cdot a \quad(a \in \mathcal{A}) .
$$

Denote by $\operatorname{Der}(X, Z)$ the set of all derivations from $X$ into $Z$ and let $\operatorname{Der}_{\delta}(X, Z)$, where $\delta: \mathcal{A} \rightarrow \mathcal{Y}$ is an ordinary derivation, be the set of all derivations from $\operatorname{Der}(X, Z)$ whose corresponding ordinary derivation is $\delta$. In particular $\operatorname{Der}_{0}(X, Z)=L_{\mathcal{A}}(X, Z)_{\mathcal{A}}$.

It is easy to see that $\operatorname{Der}(\mathcal{X}, Z)$ is a linear space: if $\Delta \in \operatorname{Der}_{\delta}(\mathcal{X}, Z)$, $\mathrm{E} \in \operatorname{Der}_{\epsilon}(\mathcal{X}, Z)$, and $\alpha, \beta \in \mathbb{C}$, then $\alpha \Delta+\beta \mathrm{E} \in \operatorname{Der}_{\alpha \delta+\beta \epsilon}(\mathcal{X}, Z)$. 
Let an $\mathcal{A}$-bimodule $\mathcal{X}$ be represented on the pair $(\mathcal{A}, \mathcal{X})$ by the regular representations $\Lambda$ and $\mathrm{P}$. The Lie product of derivations $\Delta \epsilon$ $\operatorname{Der}_{\delta}(X, X)$ and $\mathrm{E} \in \operatorname{Der}_{\epsilon}(X, X)$ is the derivation $\Delta \mathrm{E}-\mathrm{E} \Delta$, which has $\delta \epsilon-\epsilon \delta$ as the corresponding derivation.

The dual module of an $\mathcal{A}$-bimodule $\mathcal{X}$ is the dual space $\mathcal{X}^{\prime}$ with a bimodule structure that is given by

$$
\langle b \cdot \xi \cdot a, x\rangle=\langle\xi, a \cdot x \cdot b\rangle \quad\left(a, b \in \mathcal{A}, x \in \mathcal{X}, \xi \in X^{\prime}\right)
$$

(see [4], Example 2.6.2 (v)).

Proposition 3.3. Let $\mathcal{X}$ be an $\mathcal{A}$-bimodule and $(\mathcal{Y}, Z)$ a modular pair over $\mathcal{X}$ with $\Theta$ and $\mathrm{H}$ as the associated representation and antirepresentation, respectively. Then the pair $\left(\mathcal{Y}, X^{\prime}\right)$ is modular over $Z^{\prime}$.

Proof. We have to construct a right $\mathcal{A}$-modular representation and a left $\mathcal{A}$-modular antirepresentation of $\mathcal{Z}^{\prime}$ on $\left(\mathcal{Y}, X^{\prime}\right)$ in a natural way from $\Theta$ and $H$. We shall show how the antirepresentation is constructed. This is a map $\Theta^{\sharp}: Z^{\prime} \rightarrow L\left(y, X^{\prime}\right)$ defined in the following way. Define a linear map $\Theta_{y}: X \rightarrow Z$ by $\Theta_{y}(x):=$ $\Theta(x) y \quad(x \in X)$. Then, for $\zeta \in \mathcal{Z}^{\prime}$, let $\Theta^{\sharp}(\zeta)$ be given by $\Theta^{\sharp}(\zeta) y:=$ $\Theta_{y}^{\prime}(\zeta) \quad(y \in y)$. Thus

$$
\left\langle\Theta^{\sharp}(\zeta) y, x\right\rangle=\langle\zeta, \Theta(x) y\rangle \quad(x \in X, y \in \mathcal{y}, z \in \mathcal{Z}) .
$$

It is easy to see that $\Theta^{\sharp}: Z^{\prime} \rightarrow L\left(y, X^{\prime}\right)$ is a left $\mathcal{A}$-modular antirepresentation.

A similarly defined map $H^{\sharp}: Z^{\prime} \rightarrow L\left(y, X^{\prime}\right)$ is a right $\mathcal{A}$-modular representation.

If, in Proposition 3.3, $X$ is a Banach bimodule and $(y, z)$ is a Banach modular pair over $X$, then $\left(\mathcal{Y}, X^{*}\right)$ is a Banach modular pair over $Z^{*}$. The construction of the bounded antirepresentation $\Theta^{\sharp}$ and representation $\mathrm{H}^{\sharp}$ is the same as in the above proof.

Theorem 3.4. Let $\mathcal{X}$ be an $\mathcal{A}$-bimodule and $(\mathcal{Y}, \mathcal{Z})$ a modular pair over $X$. If $\Delta: X \rightarrow Z$ is a derivation with the corresponding ordinary derivation $\delta: \mathcal{A} \rightarrow \mathcal{y}$, then the adjoint map $\Delta^{\prime}: Z^{\prime} \rightarrow X^{\prime}$ is a derivation with the corresponding derivation $-\delta$.

Proof. Let $\Theta$ and $\mathrm{H}$ be the representation and the antirepresentation, respectively, of $\mathcal{X}$ on $(y, Z)$. It is straightforward to see that

$$
\Delta^{\prime}(a \cdot \zeta)=a \cdot \Delta^{\prime}(\zeta)-\Theta^{\sharp}(\zeta) \delta(a)
$$


and

$$
\Delta^{\prime}(\zeta \cdot a)=-\mathrm{H}^{\sharp}(\zeta) \delta(a)+\Delta^{\prime}(\zeta) \cdot a
$$

for all $a \in \mathcal{A}$ and $\zeta \in \mathcal{Z}^{\prime}$.

For derivations in Examples 3.2 we have the following corollary.

Corollary 3.5. (a) The adjoint $\delta^{\prime}$ of an ordinary derivation $\delta: \mathcal{A} \rightarrow$ $X$ is a derivation from $X^{\prime}$ into $\mathcal{A}^{\prime}$ with $-\delta$ as the corresponding ordinary derivation.

(b) If $\mathrm{M} \in L_{\mathcal{A}}(X, Z)_{\mathcal{A}}$, then $\mathrm{M}^{\prime} \in L_{\mathcal{A}}\left(Z^{\prime}, X^{\prime}\right)_{\mathcal{A}}$,

(c) The adjoint of an inner derivation $\Delta_{y}(x)=\Theta(x) y-\mathrm{H}(x) y$ is the inner derivation $\Delta_{y}^{\prime}(\zeta)=\Theta^{\sharp}(\zeta) y-\mathrm{H}^{\sharp}(\zeta) y$ with $-\delta_{y}$ as the corresponding derivation.

\section{Singer-Wermer Theorem}

In this section we shall confine ourselves to the case of Banach bimodules over a unital commutative Banach algebra. Our goal is to extend the well known Singer-Wermer Theorem (see [2], §18, Theorem 16) to Banach bimodules.

Recall first that the Gelfand radical of $\mathcal{A}$ is the intersection of all maximal ideals. It is well known (cf. $[2,4,6]$ ) that: (i) maximal ideals in $\mathcal{A}$ are closed and hyper maximal, i.e. their codimension is 1 ; (ii) there is a bijective correspondence between maximal ideals and characters (i.e. nonzero multiplicative linear functionals).

Following these facts we shall introduce a notion which is a counterpart of the notion of character.

Definition 4.1. Let $\mathcal{X}$ be a Banach $\mathcal{A}$-bimodule and $(\varphi, \psi)$ a pair of characters on $\mathcal{A}$. A linear functional $\xi \in X^{*}$ is a point multiplier at $(\varphi, \psi)$ if

$$
\langle\xi, a \cdot x \cdot b\rangle=\varphi(a) \psi(b)\langle\xi, x\rangle \quad(a, b \in \mathcal{A}, x \in \mathcal{X}) .
$$

Let us denote by $\Sigma_{(\varphi, \psi)}(\mathcal{X})$ the set of all point multipliers at $(\varphi, \psi)$. It is easily seen that $\Sigma_{(\varphi, \psi)}(X)$ is a weak* closed submodule in $X^{*}$. The algebra actions are very simple on $\Sigma_{(\varphi, \psi)}(X)$. For arbitrary $\xi \in$ $\Sigma_{(\varphi, \psi)}(X)$ and $a, b \in \mathcal{A}$ we have $a \cdot \xi \cdot b=\varphi(b) \psi(a) \xi$.

Example 4.2. Let $\mathcal{A}$ be considered as a Banach $\mathcal{A}$-bimodule via the regular representations. If $\varphi$ and $\psi$ are characters on $\mathcal{A}$, then $\Sigma_{(\varphi, \varphi)}(\mathcal{A})=\mathbb{C} \varphi$ and $\Sigma_{(\varphi, \psi)}(\mathcal{A})=\{0\}$, if $\varphi \neq \psi$. This follows from

$$
\varphi(a)\langle\xi, 1\rangle=\langle\xi, a\rangle=\psi(a)\langle\xi, 1\rangle \quad(a \in \mathcal{A})
$$


for $\xi \in \Sigma_{(\varphi, \psi)}(\mathcal{A})$.

For a pair of characters $(\varphi, \psi)$ on $\mathcal{A}$, let $\mathbb{C}_{(\varphi, \psi)}$ be the $\mathcal{A}$-bimodule $\mathbb{C}$ defined by

$$
a \cdot z=\varphi(a) z \quad \text { and } \quad z \cdot a=\psi(a) z \quad(a \in \mathcal{A}, z \in \mathbb{C}) .
$$

Set $\mathbb{C}_{\varphi}:=\mathbb{C}_{(\varphi, \varphi)}$. It is evident that every point multiplier $\xi \in X^{*}$ at $(\varphi, \psi)$ can be considered as a bounded representation of $\mathcal{X}$ on the pair $\left(\mathbb{C}_{\psi}, \mathbb{C}_{\varphi}\right)$.

For a submodule $\mathcal{P}$ in a Banach $\mathcal{A}$-bimodule $X$ we shall say that it is hyper maximal if it is maximal and has codimension 1 (i.e. it is a linear subspace with codimension 1 , invariant for the actions of $\mathcal{A})$.

Proposition 4.3. Let $\mathcal{X}$ be a Banach $\mathcal{A}$-bimodule. A subspace $\mathcal{P}$ in $X$ is a closed hyper maximal submodule if and only if there exists a nonzero point multiplier $\xi$ on $\mathcal{X}$ such that $\mathcal{P}=\operatorname{ker} \xi$.

Proof. Let $\mathcal{P}$ be a closed hyper maximal submodule. Choose $\xi \in X^{*}$ and $x \in \mathcal{X}$ such that $\mathcal{P}=\operatorname{ker} \xi$ and $\quad\langle\xi, x\rangle=1$. We shall prove that

$$
\varphi(a):=\langle\xi, a \cdot x\rangle \quad \text { and } \quad \psi(a):=\langle\xi, x \cdot a\rangle \quad(a \in \mathcal{A})
$$

define a pair of characters. It is evident that $\varphi$ and $\psi$ are nonzero bounded linear functionals on $\mathcal{A}$. Let $a$ and $b$ be arbitrary from $\mathcal{A}$. Since each $y \in X$ can be written as $y=\langle\xi, y\rangle x+z$ with $z \in \operatorname{ker} \xi$, we have

$$
a b \cdot x=\langle\xi, a b \cdot x\rangle x+x_{1}
$$

for some $x_{1} \in \operatorname{ker} \xi$. On the other hand, we also have

$$
a \cdot x=\langle\xi, a \cdot x\rangle x+x_{2} \quad \text { and } \quad b \cdot x=\langle\xi, b \cdot x\rangle x+x_{3}
$$

for some $x_{2}, x_{3} \in \operatorname{ker} \xi$. Multiply the latter equation by $a$ and use then the former. We get $a b \cdot x=\langle\xi, b \cdot x\rangle\langle\xi, a \cdot x\rangle x+x_{4}$, where $x_{4} \in \operatorname{ker} \xi$. It follows

$$
\varphi(a b)=\left\langle\xi,\langle\xi, b \cdot x\rangle\langle\xi, a \cdot x\rangle x+x_{4}\right\rangle=\varphi(a) \varphi(b) .
$$

It is straightforward now that $\xi \in \Sigma_{(\varphi, \psi)}(X)$. The converse direction is evident.

Theorem 4.4. Let $\mathcal{X}$ be a Banach $\mathcal{A}$-bimodule such that $\mathcal{A}$ acts topologically cyclically on both sides of $\mathcal{X}$, i.e. there exist $u$ and $v$ in $X$ such that $\mathcal{A} \cdot u$ and $v \cdot \mathcal{A}$ are dense in $X$. 
If $\xi \in X^{*}$ is a nonzero functional such that for each $x \in \operatorname{ker} \xi$ there exists a nonzero point multiplier $\xi_{x}$ with $\left\langle\xi_{x}, x\right\rangle=0$, then $\xi$ is a point multiplier.

Proof. Step 1. If $\zeta$ is a nonzero point multiplier at $(\varphi, \psi)$, then $u$ and $v$ are not in $\operatorname{ker} \zeta$.

If $u$ were in $\operatorname{ker} \zeta$, then $\langle\zeta, a \cdot u\rangle=\varphi(a)\langle\zeta, u\rangle=0 \quad(a \in \mathcal{A})$, would give $\zeta=0$, which is not the case. Similarly, $v \notin \operatorname{ker} \zeta$.

Step 2. $u, v \notin \operatorname{ker} \xi$.

If $u$ were in $\operatorname{ker} \xi$, then there would be a nonzero point multiplier $\xi_{u}$ such that $u \in \operatorname{ker} \xi_{u}$. However this is not the case, by Step 1. The proof of $v \notin \operatorname{ker} \xi$ is the same.

There is no loss of generality if we assume that

$$
\langle\xi, u\rangle=1=\langle\xi, v\rangle .
$$

Step 3. The functionals $\mu$ and $\nu$, defined by

$$
\mu(a)=\langle\xi, a \cdot u\rangle \quad \text { and } \quad \nu(a)=\langle\xi, v \cdot a\rangle \quad(a \in \mathcal{A}),
$$

are characters on $\mathcal{A}$.

It is evident that $\mu$ and $\nu$ are bounded linear functionals on $\mathcal{A}$. It follows from (4) that $\mu(1)=1=\nu(1)$. If $a \in \operatorname{ker} \mu$, then $x:=a \cdot u \in$ $\operatorname{ker} \xi$. By the assumption, there exists a nonzero point multiplier $\xi_{x}$ at $\left(\varphi_{x}, \psi_{x}\right)$, for some characters $\varphi_{x}$ and $\psi_{x}$, such that

$$
0=\left\langle\xi_{x}, a \cdot u\right\rangle=\varphi_{x}(a)\left\langle\xi_{x}, u\right\rangle .
$$

By Step 1, $\varphi_{x}(a)=0$ and therefore $a$ is not invertible (since $\varphi_{x}$ is a character). Thus, there do not exist invertible elements in ker $\mu$. By the Gleason-Kahane-Żelazko Theorem, $\operatorname{ker} \mu$ is an ideal. It follows that $\mu$ is multiplicative. The multiplicativity of $\nu$ is proven similarly.

Step 4. $\xi$ is a point multiplier at $(\mu, \nu)$.

Let $a \in \mathcal{A}$ and $x \in \mathcal{X}$ be given. If $x=b \cdot u$, then

$$
\langle\xi, a \cdot x\rangle=\langle\xi, a b \cdot u\rangle=\mu(a b)=\mu(a)\langle\xi, x\rangle .
$$

Since $\xi$ is continuous and using the hypotheses on $X$ and $\mathcal{A}$ we also have $\langle\xi, a \cdot x\rangle=\mu(a)\langle\xi, x\rangle$ for an arbitrary $x \in \mathcal{X}$. Of course, $\langle\xi, x \cdot a\rangle=$ $\nu(a)\langle\xi, x\rangle$ is proven in the same way.

The previous theorem shows that point multipliers are indeed like characters. Namely, this theorem can be considered as a module version of the famous Gleason-Kahane-Żelazko Theorem. Let $\mathcal{A}$ be a unital commutative Banach algebra and let $\mathcal{M}$ be a closed subspace in $\mathcal{A}$ with codimension 1 . Gleason and, independently, Kahane and 
Żelazko proved that $\mathcal{M}$ is an ideal if and only if it does not contain an invertible element, i.e. if each element of $\mathcal{M}$ is contained in some maximal ideal. The reader is referred to $[2,6]$ for details. According to Proposition 4.3 we can rephrase Theorem 4.4 as follows. Let $X$ be a Banach $\mathcal{A}$-bimodule such that $\mathcal{A}$ acts topologically cyclically on both sides of $\mathcal{X}$. If $\mathcal{P}$ is a closed subspace in $\mathcal{X}$ with codimension 1 such that each $x \in \mathcal{P}$ is contained in some hyper maximal submodule $\mathcal{P}_{x}$, then $\mathcal{P}$ is a submodule.

Definition 4.5. The Gelfand radical $\operatorname{rad}(X)$ of a Banach $\mathcal{A}$-bimodule $X$ is the intersection of all closed hyper maximal submodules in $X$.

We shall say that the bimodule $X$ is hyper semisimple if $\operatorname{rad}(X)=$ $\{0\}$.

Recall from [1] that the Jacobson radical of an $\mathcal{A}$-module $\mathcal{X}$ is the intersection of all maximal submodules. Hence the Jacobson radical of a Banach bimodule is always included in the Gelfand radical. However, since it is not necessarily that a maximal submodule in a Banach module is closed and hyper maximal, it is possible that the Jacobson radical is a proper subset in the Gelfand radical.

Examples 4.6. (a) The $\mathcal{A}$-bimodule $\mathbb{C}_{(\varphi, \psi)}$ is hyper semisimple. Indeed, the map

$$
\iota: \mathbb{C}_{(\varphi, \psi)} \rightarrow \mathbb{C}, \quad z \mapsto z
$$

is a nonzero point multiplier at $(\varphi, \psi)$ with the kernel $\operatorname{ker} \iota=\{0\}$.

(b) If the algebra $\mathcal{A}$ is considered as an $\mathcal{A}$-bimodule via the regular representations, then the Gelfand radical is the intersection of all maximal ideals (i.e. it is the Jacobson radical of the algebra $\mathcal{A}$.)

(c) Let $\mathcal{C}[0,1]$ be the Banach algebra of all continuous complex functions defined on the interval $[0,1]$ and let $\mathcal{C}^{1}[0,1]$ be the Banach algebra of all functions in $\mathcal{C}[0,1]$ that have continuous derivative. Then $\mathcal{C}[0,1]$ is a hyper semisimple Banach $\mathcal{C}^{1}[0,1]$-bimodule. Indeed. For $t \in[0,1]$ let $\mathcal{M}_{t}$ denote the subset of all those functions $f \in \mathcal{C}[0,1]$ that vanish at $t$. It is well known and easily to see that every $\mathcal{M}_{t}$ is a closed submodule with codimension 1 in the $\mathcal{C}^{1}[0,1]$-bimodule $\mathcal{C}[0,1]$. The assertion follows since $\cap_{t \in[0,1]} \mathcal{M}_{t}=\{0\}$.

Let $X$ be a Banach $\mathcal{A}$-bimodule and $(y, z)$ a Banach modular pair over $X$. Let $\Delta: X \rightarrow Z$ be a bounded derivation. Is the range of $\Delta$ included in the Gelfand radical of $z$ ? The answer is no as the following two examples show. 
Examples 4.7. (a) Let $\mathcal{A}$ be a unital commutative Banach algebra and $\varphi$ a character on it. Recall that a point derivation at $\varphi$ is a derivation $\delta$ that maps from $\mathcal{A}$ into the $\mathcal{A}$-bimodule $\mathbb{C}_{\varphi}$ (cf. [4], Definition 1.8.7). It is well known that there do exist algebras with nontrivial point derivations. Of course, these serve as counter examples to the above question because $\mathbb{C}_{\varphi}$ is hyper semisimple (see Example 4.6 (a)).

(b) We already know that $(\mathfrak{C}[0,1], \mathcal{C}[0,1])$ is a Banach modular pair over $\mathcal{C}^{1}[0,1]$ and that $\mathfrak{C}[0,1]$ is hyper semisimple. The derivation

$$
\mathcal{C}^{1}[0,1] \rightarrow \mathcal{C}[0,1], \quad f \mapsto f^{\prime}
$$

is bounded and nonzero, hence its range is not included in the Gelfand radical of $\mathcal{C}[0,1]$.

In order to avoid the previous examples we shall confine ourselves to the situation when $y=\mathcal{A}$. However, this is not enough.

Example 4.8. Let $X$ be a hyper semisimple Banach $\mathcal{A}$-bimodule. Then $(\mathcal{A}, X)$ is a Banach modular pair over $X$ (via the regular representations $\Lambda$ and $\mathrm{P}$ ). If $\mathrm{M}$ is a nonzero module homomorphism on $X$, then it is a derivation on $X$ whose range is not included in the Gelfand radical.

Hence module homomorphisms have to be excluded. We shall make the following additional restriction. The smallest closed submodule in $\mathcal{X}$ that contains $\operatorname{ker} \Delta$ is $\mathcal{X}$. In other words, $\mathcal{A} \cdot \operatorname{ker} \Delta \cdot \mathcal{A}$ is dense in $\mathcal{X}$. Note that every ordinary derivation $\delta$ on $\mathcal{A}$ has this property because of $1 \in \operatorname{ker} \delta$.

Theorem 4.9. Let $\mathcal{X}$ be a Banach $\mathcal{A}$-bimodule and $(\mathcal{A}, Z)$ a Banach modular pair over $X$. If $\Delta$ is a bounded derivation from $X$ into $Z$ such that $\mathcal{A} \cdot \operatorname{ker} \Delta \cdot \mathcal{A}$ is dense in $\mathcal{X}$, then its range is included in the Gelfand radical of $z$.

Proof. Let $\mathcal{X}$ be represented on $(\mathcal{A}, Z)$ by $\Theta$ and H. If $\zeta \in \Sigma_{(\varphi, \psi)}(Z)$, then

$$
\Theta(x)^{*} \zeta \in \mathbb{C} \psi \quad \text { and } \quad \mathrm{H}(x)^{*} \zeta \in \mathbb{C} \varphi \quad(x \in X) .
$$

Indeed, it is clear that, for each $x \in \mathcal{X}, \Theta(x)^{*} \zeta$ is a bounded linear functional on $\mathcal{A}$. Since

$$
\begin{aligned}
\left\langle\Theta(x)^{*} \zeta, a\right\rangle & =\langle\zeta, \Theta(x) a\rangle=\langle\zeta,(\Theta(x) 1) \cdot a\rangle \\
& =\psi(a)\left\langle\Theta(x)^{*} \zeta, 1\right\rangle \quad(a \in \mathcal{A})
\end{aligned}
$$

the assertion follows. 
Let $\delta: \mathcal{A} \rightarrow \mathcal{A}$ be the ordinary derivation that corresponds to $\Delta$. For arbitrary $a, b \in \mathcal{A}$ and $x \in \operatorname{ker} \Delta$ we have

$$
\begin{aligned}
\Delta(a \cdot x \cdot b) & =a \cdot \Theta(x) \delta(b)+a \cdot \Delta(x) \cdot b+\mathrm{H}(x) \delta(a) \cdot b \\
& =a \cdot \Theta(x) \delta(b)+\mathrm{H}(x) \delta(a) \cdot b
\end{aligned}
$$

and therefore, for an arbitrary point multiplier $\zeta \in \Sigma_{(\varphi, \psi)}(\mathcal{Z})$,

$$
\langle\zeta, \Delta(a \cdot x \cdot b)\rangle=\varphi(a)\left\langle\Theta(x)^{*} \zeta, \delta(b)\right\rangle+\psi(b)\left\langle\mathrm{H}(x)^{*} \zeta, \delta(a)\right\rangle=0,
$$

by the Singer-Wermer Theorem. Since $\mathcal{A} \cdot \operatorname{ker} \Delta \cdot \mathcal{A}$ is dense in $X$ and since $\Delta$ is linear and continuous it follows, by Proposition 4.3 , that $\Delta(X) \subseteq \operatorname{rad}(Z)$.

\section{REFERENCES}

[1] F. Anderson and K. Fuller, Rings and Categories of Modules, Graduate Texts in Mathematics 13, Springer-Verlag, New York (1974).

[2] F. F. Bonsal and J. Duncan, Complete Normed Algebras, Springer, Berlin (1973).

[3] M. BREŠAR, On the distance of the composition of two derivations to the generalized derivations, Glasgow Math. J. 33 (1991), 89-93.

[4] H. G. Dales, Banach Algebras and Automatic Continuity, London Mathematical Society Monographs, Vol. 24, Clarendon Press, Oxford (2000).

[5] H. G. Dales, A. Rodriguez-Palacios, and M. V. Velasco, The second transpose of a derivation, J. London Math. Soc., (2), 64, (2001), 707-721.

[6] T. W. Palmer, Banach Algebras and the General Theory of $*$-Algebras, Volume 1: Algebras and Banach Algebras, Encyclopedia of Mathematics and its Applications 49, Cambridge University Press (1994).

Janko Bračič,

University of Ljubljana,

IMFM,

Jadranska 19,

1111 Ljubljana, Slovenia

janko.bracic@guest.arnes.si

Received on 24 August 2001 and in revised form on 28 November 2001. 